ARTICLE OPEN

\title{
Imaging the formation and surface phase separation of the
}

\section{$C E$ phase}

\author{
Haibiao Zhou (iD) ${ }^{1,2,6 凶}$, Qiyuan Feng ${ }^{1,2}$, Yubin Hou ${ }^{1}$, Masao Nakamura (iD) ${ }^{3}$, Yoshinori Tokura (iD ${ }^{3,4}$, Masashi Kawasaki ${ }^{3,4}$, \\ Zhigao Sheng $\mathbb{D}^{1,2,5 \bowtie}$ and Qingyou Lu (iD) ${ }^{1,2,5 \bowtie}$
}

The $C E$ phase is an extraordinary phase exhibiting the simultaneous spin, charge, and orbital ordering due to strong electron correlation. It is an ideal platform to investigate the role of the multiple orderings in the phase transitions and discover emergent properties. Here, we use a cryogenic high-field magnetic force microscope to image the phase transitions and properties of the CE phase in a $\operatorname{Pr}_{0.5} \mathrm{Ca}_{0.5} \mathrm{MnO}_{3}$ thin film. In a high magnetic field, we observed a clear suppression of magnetic susceptibility at the charge-ordering insulator transition temperature $\left(T_{\mathrm{CO}}\right)$, whereas, at the Néel temperature $\left(T_{\mathrm{N}}\right)$, no significant change is observed. This observation favors the scenario of strong antiferromagnetic correlation developed below $T_{\mathrm{CO}}$ but raises questions about the Zener polaron paramagnetic phase picture. Besides, we discoverd a phase-separated surface state in the $C E$ phase regime. Ferromagnetic phase domains residing at the surface already exist in zero magnetic field and show ultra-high magnetic anisotropy. Our results provide microscopic insights into the unconventional spin- and charge-ordering transitions and revealed essential attributes of the $C E$ phase, highlighting unusual behaviors when multiple electronic orderings are involved.

npj Quantum Materials (2021)6:56; https://doi.org/10.1038/s41535-021-00353-2

\section{INTRODUCTION}

Strong electron correlation in materials usually leads to complex and emergent phenomena ${ }^{1}$, which provide a fertile ground for exploring new physics and hold great potential for applications. The colossal magnetoresistance in perovskite manganites and unconventional high- $T_{C}$ superconductivity in cuprates are the two most distinguished phenomena in strongly correlated oxides. These materials share many common characteristics. For example, both of them exhibit a variety of phases with contrasting properties. In manganites, there exist the well-known ferromagnetic metallic (FM) phase, antiferromagnetic (AFM) charge-ordered insulating (COI) phase, and so on. Among them, the CE phase, first proposed by Goodenough in the $1950 \mathrm{~s}^{2}$, is most prominent as it is an exceptional realization of simultaneous ordering of spin, charge, and orbital. How the phase transitions and properties are affected in the presence of multiple orderings is a pivotal question in strongly correlated materials. Hence, the $C E$ phase, existing in narrow-bandwidth half-doped manganites, such as $R E_{0.5} \mathrm{Ca}_{0.5} \mathrm{MnO}_{3} \quad(R E=\mathrm{Pr}, \mathrm{Sm}, \mathrm{Y}, \mathrm{Nd} \text {, etc })^{3-7}$ and single-layered manganite $\mathrm{La}_{0.5} \mathrm{Sr}_{1.5} \mathrm{MnO}_{4}{ }^{8}$, is an ideal platform to investigate these effects 9 .

These half-doped manganites show a similar phase diagram as in Fig. $1 \mathrm{a}$, which is for bulk $\operatorname{Pr}_{1-x} \mathrm{Ca}_{x} \mathrm{MnO}_{3}{ }^{3,4}$. When close to half doping $(x \sim 0.5)$, before entering the $C E$ phase, it first undergoes a transition from paramagnetic (PM) phase to charge/orbitalordered insulating phase at a charge-ordering insulator transition temperature $\left(T_{\mathrm{COI}}\right)=240 \mathrm{~K}$, then followed by an AFM transition at Néel temperature $\left(T_{N}\right)=175 \mathrm{~K}$. Below $T_{N}$ it shows the $C E$-type charge, spin and orbital ordering (Fig. 1b) ${ }^{2}$. This $C E$ phase features the ferromagnetic zigzag chains consisting of $\mathrm{Mn}^{3+} / \mathrm{Mn}^{4+}$ ions with alternating $d_{3 x^{2}-r^{2}}$ and $d_{3 y^{2}-r^{2}}$ orbitals at the $\mathrm{Mn}^{3+}$ ion sites, driven by the competition between the electron kinetic energy and magnetic exchange energy ${ }^{10,11}$. Besides, it has an equal number of $\mathrm{Mn}^{3+}$ and $\mathrm{Mn}^{4+}$ cations, resulting in a concomitant charge ordering with a checkboard pattern. Owing to the enhanced tendency towards the COI state at this doping level, the $C E$ phase possesses a high melting magnetic field and shows the largest colossal magnetoresistive effect. For example, a magnetic field larger than $30 \mathrm{~T}$ is needed to transform this phase into the FM phase in bulk $\operatorname{Pr}_{0.5} \mathrm{Ca}_{0.5} \mathrm{MnO}_{3}{ }^{12}$, which is, however, inaccessible to most investigating tools.

Although this phase has attracted intensive interest, several mysteries remain to be fully solved. A thorough investigation of these questions is crucial to our understanding of strongly correlated systems. One of them is how the CE phase is formed. Unlike many other manganites where spin and charge orderings occur at the same temperature, the CE phase is attained after the successive COI and AFM transitions. But, the drop of magnetization, expected to occur at $T_{\mathrm{N}}$, appears at $T_{\mathrm{CO}}$ instead. Although at $T_{N}$, no well-defined signal has been detected (Fig. 1c). The nature of the magnetic anomaly at $T_{\mathrm{CO}}$, as well as the magnetic structure of the intermediate phase between $T_{\mathrm{CO}}$ and $T_{\mathrm{N}}$, has been a longlasting puzzle. This is common to the aforementioned half-doped manganites. At present, there are mainly two scenarios proposed for this discrepancy. In the one model, which we call short-range AFM correlation scenario, the decrease in magnetization at $T_{\mathrm{CO}}$ has been attributed to the quenching of double-exchange interaction and onset of AFM correlation, due to the localization of the charge carriers ${ }^{8,13-15}$. Short-range antiferromagnetically coupled zigzag chain fragments start to form at this temperature, and the smooth increase of magnetic correlation across $T_{N}$ is ascribed to the coherent ordering of the zigzag chains ${ }^{15}$. However, the evidence

\footnotetext{
${ }^{1}$ Anhui Key Laboratory of Condensed Matter Physics at Extreme Conditions, High Magnetic Field Laboratory, HFIPS, Anhui, Chinese Academy of Sciences, Hefei, China. ${ }^{2}$ Hefei National Laboratory for Physical Sciences at the Microscale and Anhui Laboratory of Advanced Photon Science and Technology, University of Science and Technology of China, Hefei, China. ${ }^{3}$ RIKEN Center for Emergent Matter Science (CEMS), Wako, Saitama, Japan. ${ }^{4}$ Department of Applied Physics and Quantum Phase Electronics Research Center (QPEC), University of Tokyo, Tokyo, Japan. ${ }^{5}$ Collaborative Innovation Center of Advanced Microstructures, Nanjing University, Nanjing, China. ${ }^{6}$ Present address: Department of Condensed Matter Physics, Weizmann Institute of Science, Rehovot, Israel. ${ }^{凶}$ email: haibiaozhou24@gmail.com; zhigaosheng@hmfl.ac.cn; qxl@ustc.edu.cn
} 

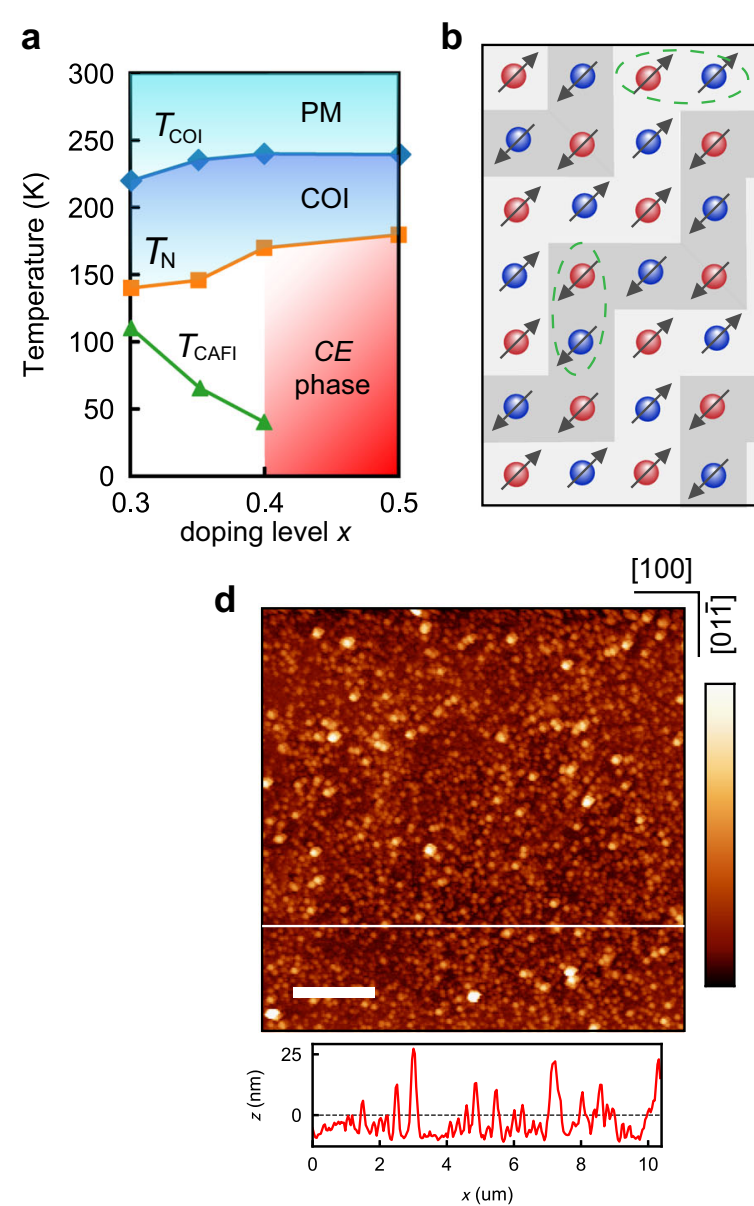
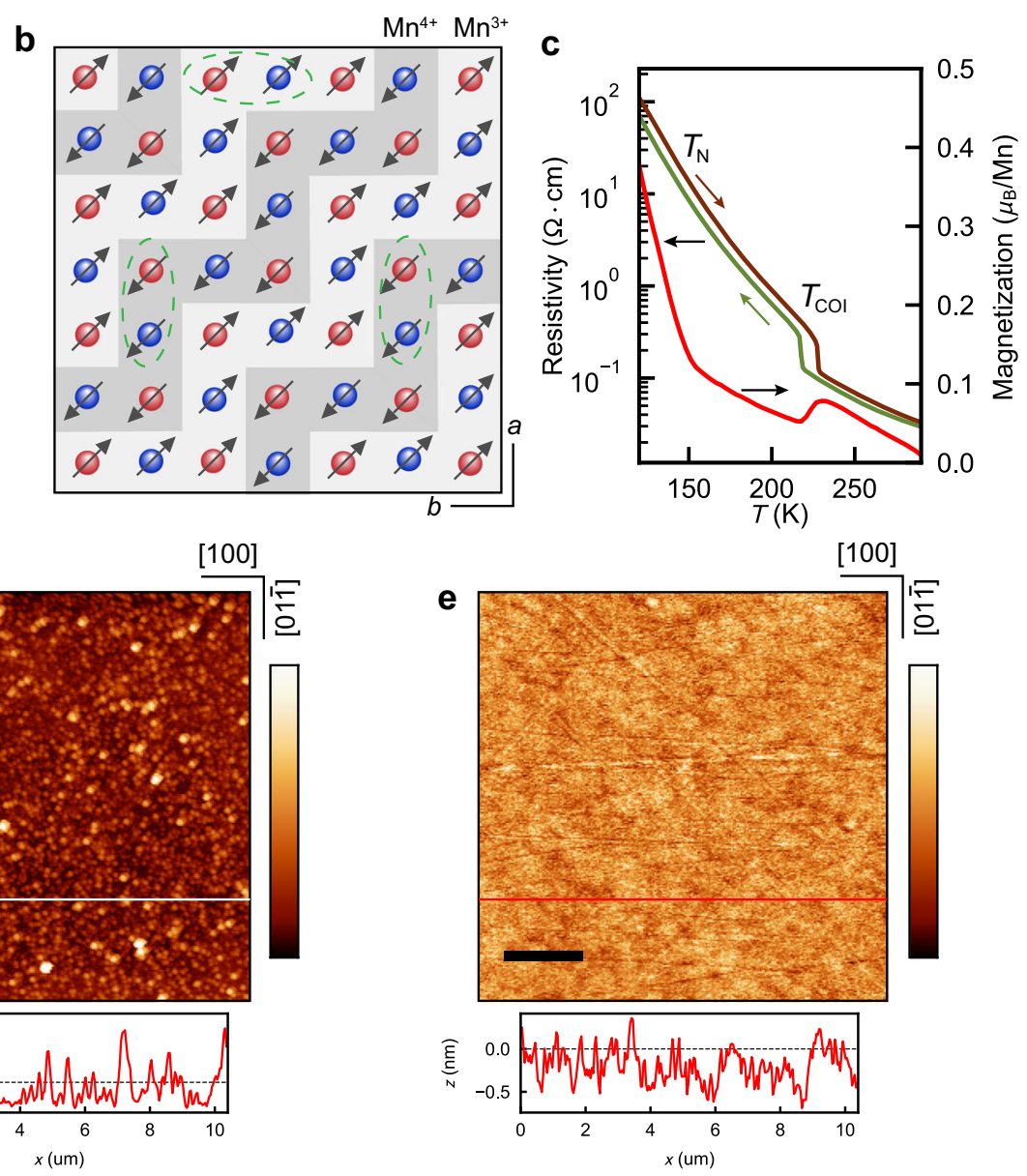

Fig. 1 Phase diagram, electrical, and magnetic properties of PCMO. a Phase diagram of $\operatorname{Pr}_{1-x} \mathrm{Ca}_{x} \mathrm{MnO}_{3}$ as a function of the doping level $x$ adapted from ref. ${ }^{4}$. PM, COI, and CAFI denote the paramagnetic insulator, charge-ordered insulator, and canted antiferromagnetic insulator phase, respectively. $\mathbf{b}$ Schematic illustration of the charge and spin ordering in the CE phase. The ferromagnetic zigzag chains are shown by the gray and light gray zigzag regions. The green-dashed ovals indicate the Zener polarons. $\mathbf{c}$ The temperature dependence of the resistivity and magnetization of the imaged PCMO film. The resistivity was measured in zero field and the magnetization was measured in $0.1 \mathrm{~T}$ applied along [100] axis with the diamagnetic response of the substrate subtracted. d-e Rough and flat topography observed in this film at different locations. Image size: $10.4 \times 10.4 \mu \mathrm{m}^{2}$. Scale bar in $\mathbf{d}$ and e: $2 \mu \mathrm{m}$. Color scale: $60 \mathrm{~nm}$ (d) and $2.2 \mathrm{~nm}(\mathbf{e})$.

\begin{tabular}{|c|c|c|c|c|c|c|c|c|}
\hline \multirow[t]{2}{*}{$T_{\mathrm{COI}}$} & 0 & $x+x+x+4 x+x \rightarrow x+4$ & None & 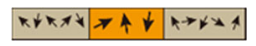 & None & $x+k x+|x k x+x| k \rightarrow x+1$ & None & \\
\hline & Low & $A \uparrow A R A \uparrow \uparrow \pi \uparrow+A R \rightarrow A \uparrow$ & None & 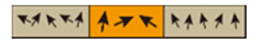 & Weak & $|x+x<1| x \times 4+4 \mid x+414$ & Weak & Weak \\
\hline
\end{tabular}

The rectangular regions, filled with arrows and different colors represent the different phases. The gray, red, orange, and green regions are the paramagnetic, $C E$, Zener polaron PM, and short-range AFM-correlated phases, respectively.

has been very limited to neutron scattering experiments ${ }^{14,15}$. The other one which is based on the formation of Zener polarons (ZP) has also gained much support after the seminal work of DaoudAladine et al. ${ }^{5,16-18}$. In contrast to the ideal site-centered checkboard pattern expected in the $C E$ model, in this picture, the ferromagnetic $\mathrm{Mn}^{3+}-\mathrm{Mn}^{4+}$ dimers, so-called $\mathrm{ZP}$, start to form at $T_{\text {COI }}$ mediated by the oxygen atom (Fig. $1 \mathrm{~b}$ ), resulting in a PM phase consisting of ZP below $T_{\mathrm{COI}}$. Suppression of the magnetization is then expected as a consequence of the paring of $\mathrm{Mn}-\mathrm{Mn}$ ions. The magnetic moments are doubled in magnitude but halved in number in the ZP PM phase ${ }^{5}$. The associated ferroelectric ordering due to inversion symmetry breaking has been reported in various manganites later ${ }^{16,17,19-21}$. It is worth noting that though it is highly demanding, no real-space microscopic imaging results 

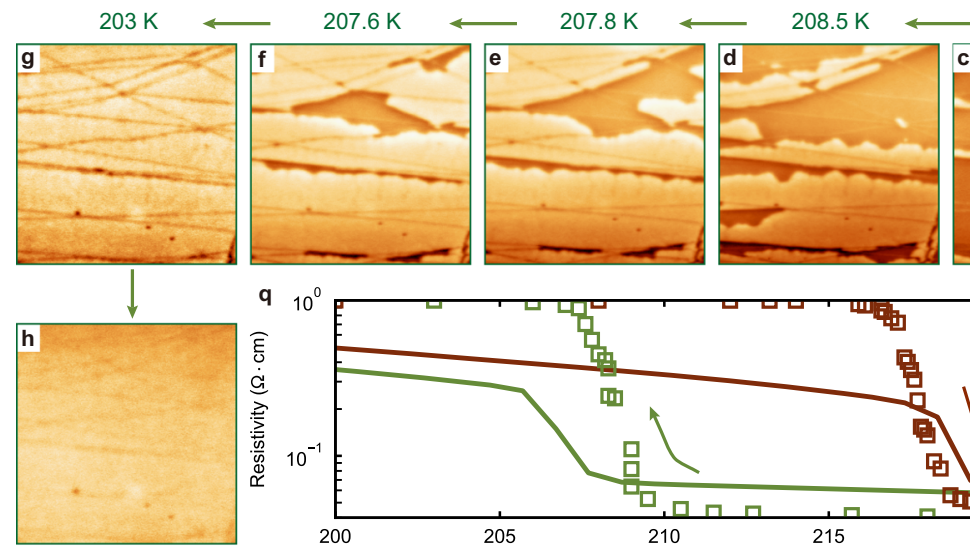

209 K

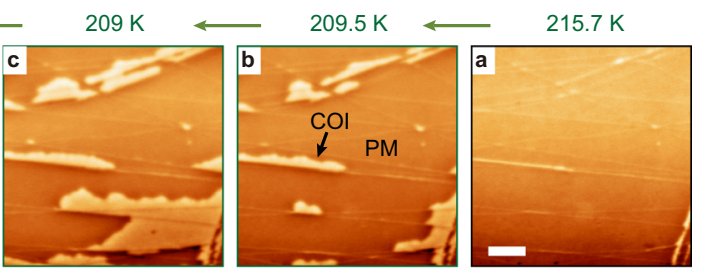

$215.7 \mathrm{~K}$

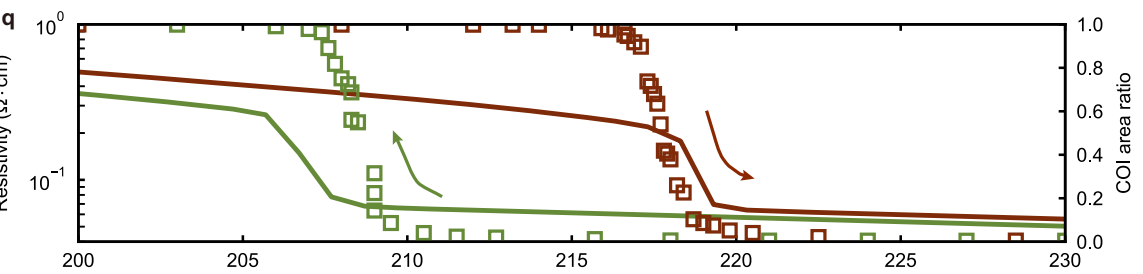

$183 \mathrm{~K}$
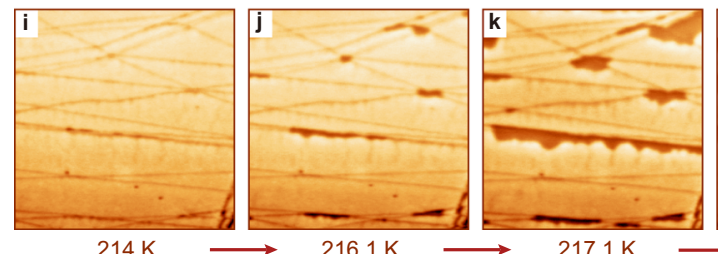

$T(\mathrm{~K})$

$214 \mathrm{~K}$

$216.1 \mathrm{~K}$

$217.1 \mathrm{~K}$

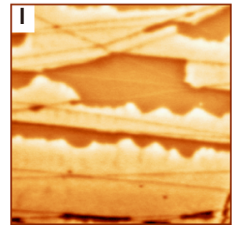

$217.3 \mathrm{~K}$
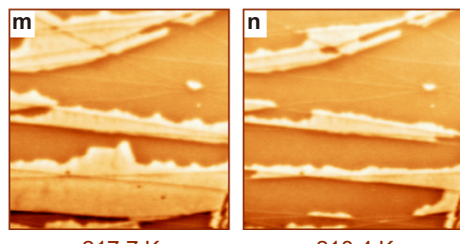

$218.4 \mathrm{~K}$

$228.5 \mathrm{~K}$
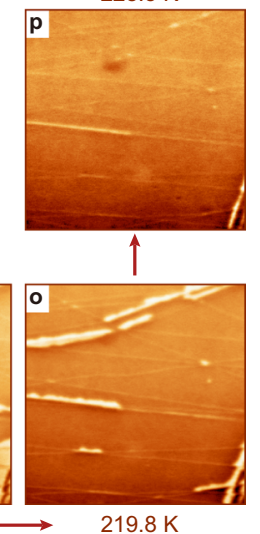

Fig. 2 Imaging the magnetic contrast at $T_{\mathrm{col}}$. The magnetic images are taken on a rough region while the sample is cooled down from room temperature to $183 \mathrm{~K}(\mathbf{a}-\mathbf{h})$ and then warmed up to $230 \mathrm{~K}(\mathbf{i}-\mathbf{p})$ in a field of $9 \mathrm{~T}$. The arrows indicate the imaging sequence. $\mathbf{q}$ shows the temperature dependence of resistivity in $9 \mathrm{~T}$ (solid lines) and the area ratio of the charge-ordering insulating (COI) phase (open squares) extracted from the MFM images. Scale bar in a: $2 \mu \mathrm{m}$. MFM image size: $11.8 \times 11.8 \mu \mathrm{m}^{2}$. The contrast is $\sim 0.5 \mathrm{~Hz}$ for all the images.

have been reported so far for the transitions at $T_{\mathrm{COI}}$ and $T_{\mathrm{N}}$, mainly limited by the required high magnetic field. Microscopic evidence would be highly beneficial to this debate $e^{22-28}$ and also the ongoing controversy of site-centered or bond-centered charge ordering in transition metal oxides ${ }^{25,29,30}$. In this work, we show that it is possible to distinguish these scenarios through magnetic imaging in high magnetic fields, as they would give rise to very different magnetic contrasts.

Another intriguing phenomenon is the variation of the CE-type ordering when the size or dimension of the sample is reduced. Magnetic hysteresis has been widely observed in half-doped manganite nanoparticles ${ }^{31}$ and nanowires ${ }^{32}$, which is evidence of an FM phase or superparamagnetic phase. Motivated by this, the polar surfaces of half-doped manganites in the CE phase regime have been predicted to possess a unique state, arising from a deviating charge density $\left(n_{\mathrm{e}}=0.75\right)$ at the surface layer from half doping. It is neither a pure $\mathrm{COI}$ phase nor an entire FM phase. Rather, it is a PS state, i.e., a coexistence of the two phases at the surface $^{33}$. This PS surface state in the CE phase regime, however, still lacks direct evidence. Interestingly, an AFM state at the surface of FM manganites has been observed, which is attributed to the enhanced spin-orbit coupling at the surface ${ }^{34}$. More generally, it is still an open question whether there exists a bulk-surface correspondence in the magnetic or electronic states in strongly correlated systems. Hence, it is of crucial importance to verify this prediction and further reveal the surface characteristics, and magnetic imaging has been demonstrated as an ideal tool to study magnetic phase separation.

In this article, we report the real-space magnetic imaging of the magnetic transitions at $T_{\mathrm{CO}}$ and $T_{\mathrm{N}}$, and the $C E$ phase in a $\mathrm{Pr}_{0.5} \mathrm{Ca}_{0.5} \mathrm{MnO}_{3}$ (PCMO) thin film. The 30-nm thick PCMO thin film was grown on a $\left(\mathrm{LaAlO}_{3}\right)_{0.3}\left(\mathrm{SrAl}_{0.5} \mathrm{Ta}_{0.5} \mathrm{O}_{3}\right)_{0.7}$ (LSAT) (011) substrate using pulsed laser deposition (see Methods). The PCMO/LSAT (011) film (in pseudocubic notation) shows similar charge, orbital, and magnetic transitions with the bulk sample, but with reduced transition temperatures $\left(T_{\mathrm{CO}}=210 \mathrm{~K} \text { and } T_{\mathrm{N}}=150 \mathrm{~K}\right)^{35,36}$ and melting field ${ }^{37}$. The magnetic force microscope (MFM) equipped with a $20 \mathrm{~T}$ superconducting magnet used in this work has been described in detail elsewhere ${ }^{38,39}$. In brief, the contrast in MFM images comes from the long-range magnetic force between the sample and the magnetic tip. We emphasize that in the present work, the accessible high magnetic field brings us manifold advantages. First, owing to the enhanced contrast in high magnetic fields, one is capable of imaging the magnetic susceptibility change even when only weakly magnetic phases are involved ${ }^{40}$, which is crucial for the present work. Second, in high fields, very different contrast is expected for the two scenarios proposed for the magnetic anomaly at $T_{\mathrm{CO}}$, which can be directly compared with the collected MFM images. Third, this will enable us to reveal some important high-field features of the surface magnetic structures.

\section{RESULTS AND DISCUSSION}

\section{Magnetic images at $T_{\mathrm{COI}}$ and $T_{\mathrm{N}}$}

Before presenting the magnetic images, we summarize in Table 1 the image contrasts expected for a conventional PM to AFM transition and the cases of the above two scenarios. Generally, both PM and AFM phase shows zero net magnetic moments in zero field, but PM phase will show large magnetization in a strong magnetic field. Hence, in the case of a conventional PM to AFM phase transition, one should only see the image contrast during the phase domain formation in a high magnetic field at $T_{N}$, but not at $T_{\mathrm{CO}}$. In the ZP picture, one should be able to see a reduced susceptibility at $T_{\mathrm{CO}}$ as the ZP PM phase has slightly smaller susceptibility ${ }^{5}$, but a much larger contrast at $T_{N}$ should also be observed when it enters the AFM phase in a high field. However, in the short-range AFM correlation picture, short-range AFM domains start to form at $T_{\mathrm{CO}}$, and one would be able to see an obvious domain formation at $T_{\mathrm{CO}}$, whereas at $T_{\mathrm{N}}$ there should be little or no change depending on whether we can see the domain walls between the short-range zigzag chains. The strikingly different contrasts under high magnetic fields for these pictures highlight the advantage of high-field magnetic imaging. In principle, macroscopic magnetization measurements should also be able to reveal these differences. However, these measurements (including Fig. 1c) usually see a PM behavior even below $T_{N}$, which 

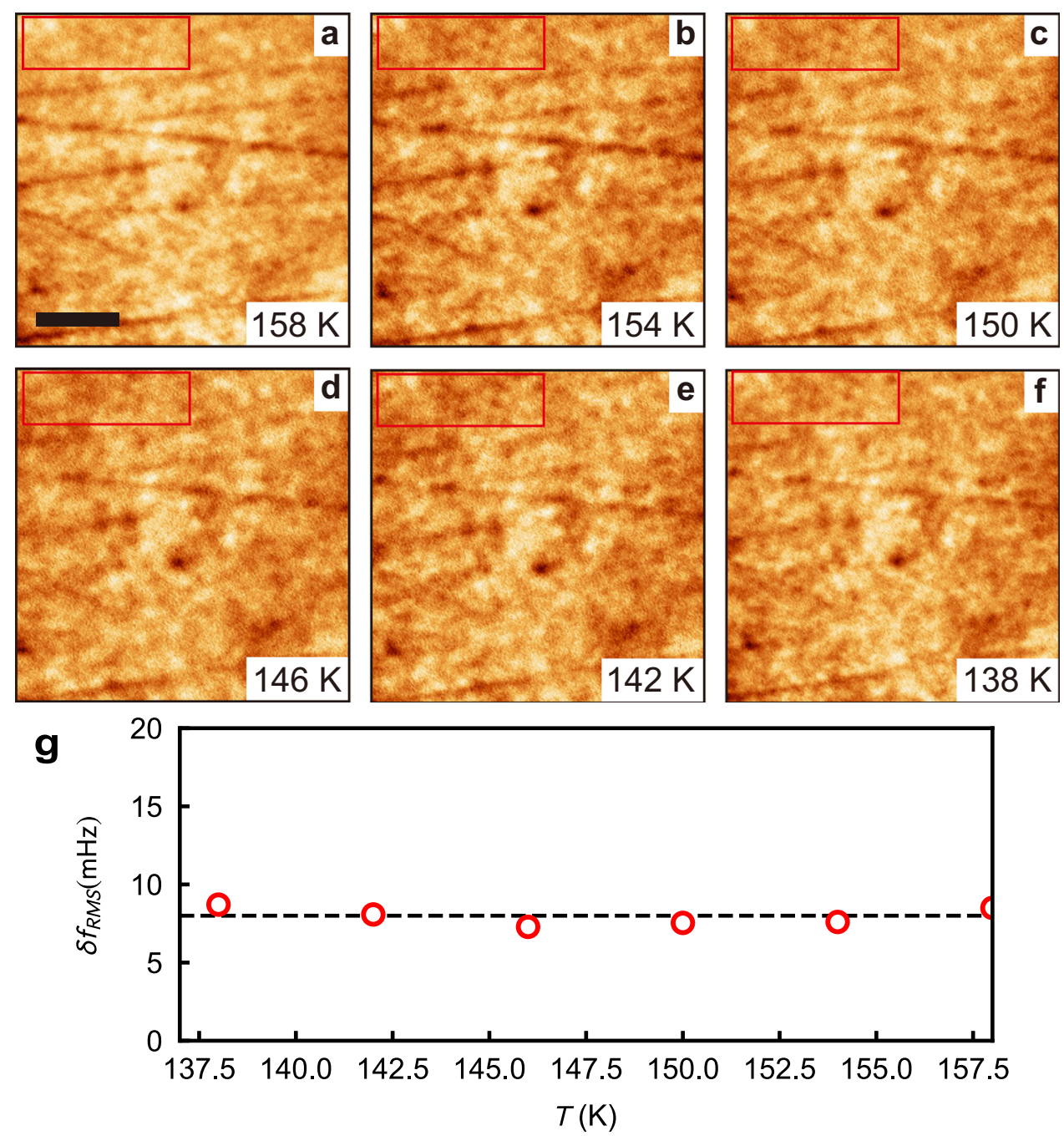

Fig. 3 Absence of significant change when the sample is cooled down through $\boldsymbol{T}_{\mathbf{N}}$. a-f MFM images taken on a rough region while the film is field-cooled through $T_{N}=150 \mathrm{~K}$ from room temperature in $9 \mathrm{~T}$. Scale bar in a: $1 \mu \mathrm{m}$. Image size: $4 \times 4 \mu \mathrm{m}^{2}$. $\mathbf{g}$ The root-mean-square (RMS) of the magnetic contrast in the region marked by the dashed red rectangle in the images of a. The color scale is $75 \mathrm{mHz}$ for all the MFM images.

is generally ascribed to impurities ${ }^{5,11,15,36}$. Local magnetic imaging microscopy would spare us their contribution.

We observed two types of surface on this single PCMO film with MFM: one is rough and covered with nanosized particles (Fig. 1d), which might be $\mathrm{MnO}_{\mathrm{x}}$, whereas the other one is very flat but shows several crosshatching lines (Fig. 1e), similar to other manganite films owing to the presence of epitaxial strain ${ }^{41}$. It will be shown that the crosshatching lines are also present underneath those particles on the rough region. These lines act as the nucleation sites of the phase domains. The root-mean-square roughness of the two-type surfaces is $\sim 10 \mathrm{~nm}$ and $3.5 \AA$, respectively. The reason for the formation of two types of surface is unclear yet.

In Figs. 2 and 3, we show the MFM images of the sample when it is cooled down in $9 \mathrm{~T}$ field through $T_{\mathrm{CO}}$ and $T_{\mathrm{N}}$, respectively. At $215.7 \mathrm{~K}$, some bright stripes residing close to the crosshatching lines are readily observed. As the temperature is going down, these stripes expand and gradually occupy the imaged area $(183 \mathrm{~K})$. Then when the sample is warmed up, the phase with darker contrast nucleates and expands, with the brighter phase domains shrinking towards the line defects. The dark phase domains dominate the sample at above $219 \mathrm{~K}$. The hysteresis undoubtedly indicates it is a first-order phase transition, and the temperature dependence of the area portion of the bright regions matches very well with that of the measured resistance. Thus, the dark and white regions are ascribed to the PM and COI phases, respectively. The brighter contrast of the $\mathrm{COI}$ phase suggests it has a smaller attractive force with the magnetic tip, thus a weaker magnetic susceptibility than the PM phase. The image contrast is greatly enhanced in $9 \mathrm{~T}$ compared with $0.5 \mathrm{~T}$ (Supplementary Figure 1). When the sample is cooled through $T_{N}$, no noticeable change is observed in the MFM images (Fig. 3 and Supplementary Figure 2). Note that the imaged area has a rough surface.

Hence, these images can be well explained by the short-range AFM correlation scenario (Table 1). In these half-doped manganites, there exist the competing FM correlation and CE-type AFM correlation. At $T_{\mathrm{CO}}$, electron localization suppresses the doubleexchange FM interaction, whereas enhances the CE-type AFM correlation $^{8,13,15}$. Short-range zigzag chains that are antiferromagnetically coupled start to form at $T_{\mathrm{CO}}$, as revealed by previous neutron scattering experiments performed in the nearly half-doped $\operatorname{Pr}_{0.55}\left(\mathrm{Ca}_{0.8} \mathrm{Sr}_{0.2}\right)_{0.45} \mathrm{MnO}_{3}{ }^{14}$, and half-doped single-layer manganite $\mathrm{La}_{0.5} \mathrm{Sr}_{1.5} \mathrm{MnO}_{4}{ }^{15,25}$. This causes the susceptibility drop seen in the macroscopic magnetization and MFM images. The AFM correlation length increases gradually when the sample enters the $C E$ phase, suggesting the short-range chain fragments merge into larger domains when the temperature is lowered ${ }^{14,15}$. The fact that the spin ordering nucleates at $T_{\mathrm{CO}}$, but its coherent ordering occurs only at $T_{N}$, implies unconventional phase dynamics ${ }^{14,42}$, perhaps a unique 

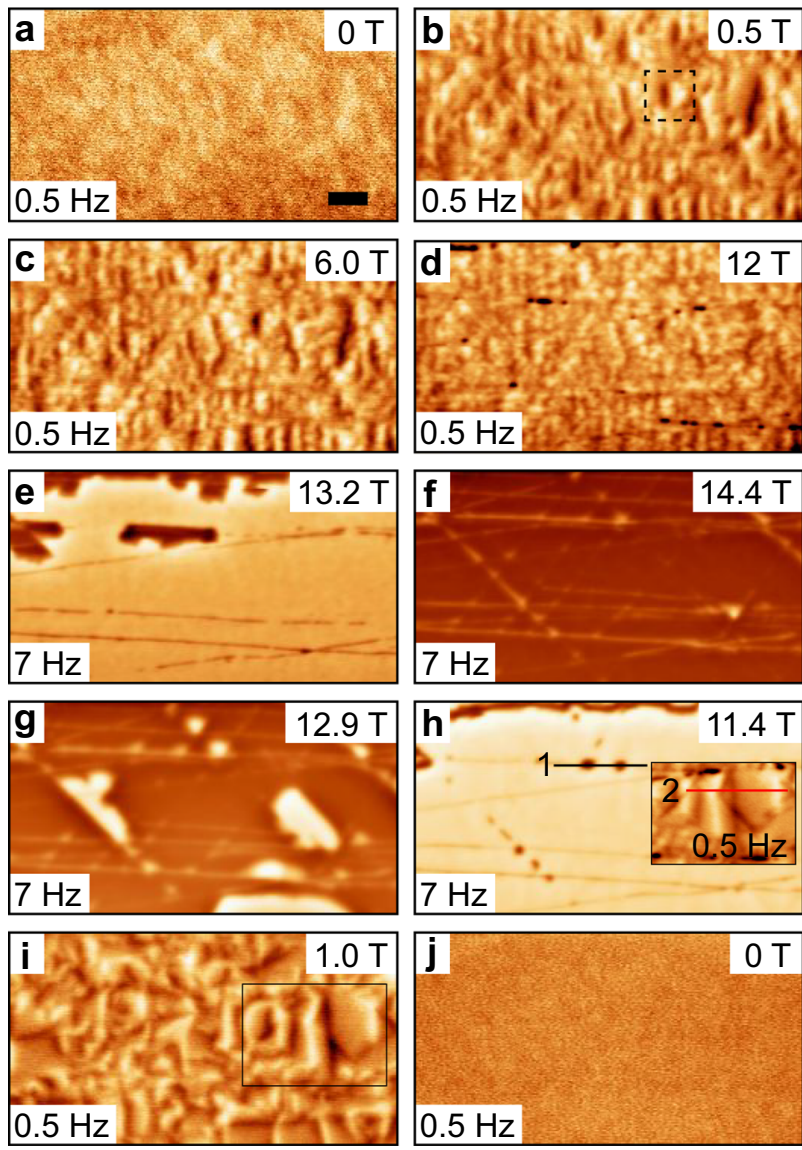
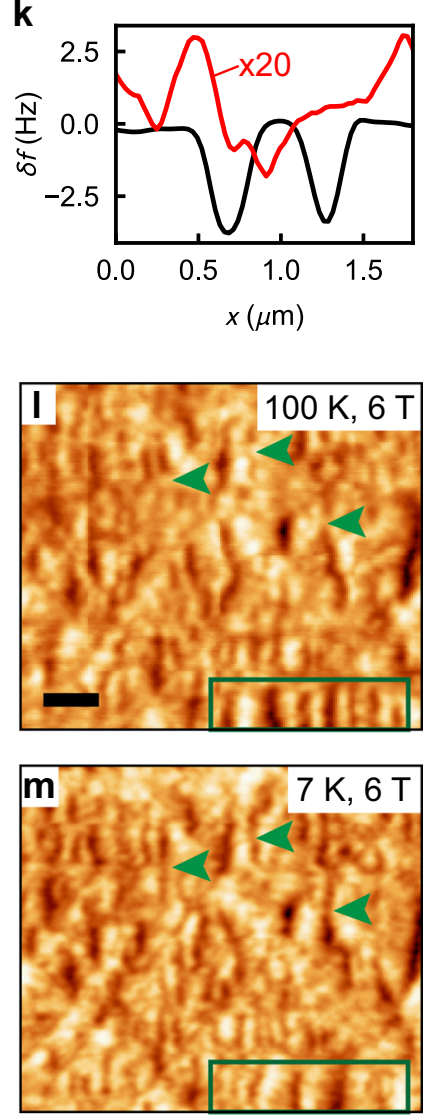

Fig. 4 Magnetic images of surface phase separation. a-j MFM images taken on a flat region in different magnetic fields at $100 \mathrm{~K}$, with the color scales labeled. The dashed rectangle in $\mathbf{b}$ contains a pair of dark and bright contrast. The inset in $\mathbf{h}$ shows the area with a reduced color scale $(0.5 \mathrm{~Hz})$ to highlight the weakly magnetic features. Scale bar in a: $1 \mu \mathrm{m}$. Image size: $11 \times 6 \mu \mathrm{m}^{2}$. $\mathbf{k}$ The line profiles taken along the two lines marked in $\mathbf{h}$. The data for the red line is multiplied by a factor of 20 . I- $\mathbf{m}$ A zoom-in view of the patterns after the sample is cooled down to $100 \mathrm{~K}(\mathbf{I})$ and $7 \mathrm{~K}(\mathbf{m})$ in different thermal cycles. Scale bar in I: $1 \mu \mathrm{m}$. Image size: $7 \times 6 \mu \mathrm{m}^{2}$. A magnetic field of $6 \mathrm{~T}$ is later applied to enhance the contrast. The arrows and frames indicate the changes of the domains.

feature of the CE-type ordering ${ }^{15}$. However, we do not observe the evolution of the short-range domains between $T_{\mathrm{COI}}$ and $T_{\mathrm{N}}$, implying the $\mathrm{COI}$ phase domains are all occupied with the short-range zigzag chain segments and the separation between the domains is smaller than the MFM spatial resolution $(\sim 30 \mathrm{~nm})$. It is worth noting that the suppression of magnetic susceptibility is also observed at the chargeordering transition temperature in some other non-half-doped manganites $\mathrm{Bi}_{0.18} \mathrm{Ca}_{0.82} \mathrm{MnO}_{3}{ }^{43}$ and some nickelates ${ }^{44}$, which may also be driven by a similar mechanism. That said, our results would also be consistent with the Zener polaron picture, as long as the strong AFM correlation of the ZP is also involved.

\section{Phase-separated surface state in the CE phase regime}

We indeed see some prominent changes on the flat region when the sample enters the $C E$ phase at $T_{\mathrm{N}}$ (Supplementary Figure 3 ). We imaged the formation of some local FM features. Nevertheless, these features are not related to the field-induced COI to FM transition as they are already present in zero field ${ }^{37}$.

To show more details of the local FM structures, in Fig. 4, we present a series of MFM images taken in a magnetic field swept between 0 and $14.4 \mathrm{~T}$ at $100 \mathrm{~K}$ after the sample is cooled down in zero field. The zero-field image already shows some faint mist-like contrast, which is significantly enhanced by the application of $0.5 \mathrm{~T}$ field, where fine stripe-like features elongated along the vertical direction ([01-1] axis) are observed. A pair of bright and dark regions as indicated by a dashed line box evidences an inplane magnetic easy axis. However, these features show little change as we increase the magnetic field except that the magnitude of contrast is enhanced, even until the CE phase starts to melt, as seen when the large FM domains appear (Fig. 4e). When the magnetic field is ramped down, the $C E$ phase reappears (Fig. 4g). Interestingly, there also exist such FM features inside the $C E$ phase domains, which are highlighted with a reduced color scale in the rectangular region in Fig. $4 \mathrm{~h}$. The domain pattern is now less anisotropic, with a shape strongly different from the case before the CE phase is melted (see Fig. $4 \mathrm{e}$ and Supplementary Figure 4). The pattern does not change when the magnetic field is further decreased (Fig. 4i), suggesting they are also pinned, similar to the field sweep-up process. In Fig. $4 \mathrm{I}-\mathrm{m}$, the images taken at $7 \mathrm{~K}$ and $100 \mathrm{~K}$ for two different thermal cycles are shown. The patterns are almost the same, and one can readily see how the small domains merge when the temperature is lowered.

We argue that the observations fully agree with the aforementioned numerical simulations based on the two-orbital doubleexchange model when taking into account the polar surface of $\mathrm{Pr}_{0.5} \mathrm{Ca}_{0.5} \mathrm{MnO}_{3}{ }^{33}$. First, the magnetic domains appear at $\sim 150 \mathrm{~K}$, suggesting that it is related to the AFM transition and CE phase. Second, the fact that the FM phase domains are much more evident on the flat region implies that it is related to the surface (Supplementary Figure 5). Third, the magnetic contrast of these features is $\sim 20$ times smaller than that between the COI and FM phases (Fig. 4l), indicating they are due to some magnetic structures that are not homogeneous through the whole film thickness. In addition, as all the low-field FM domains possess a 

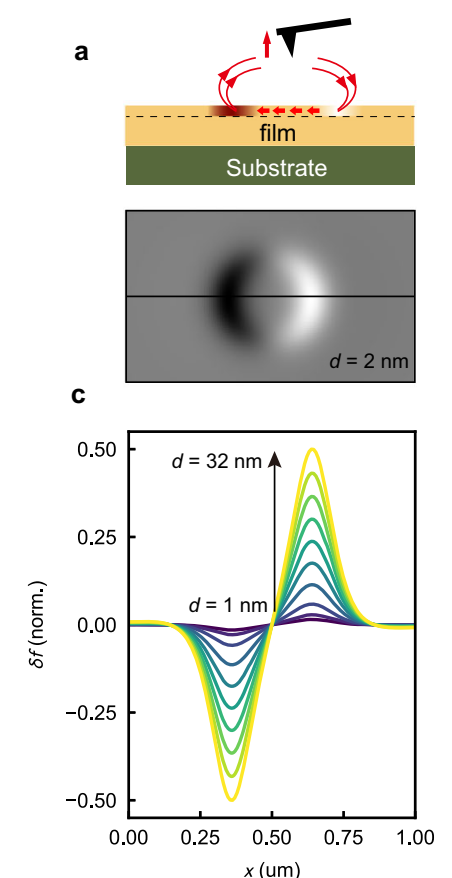

e
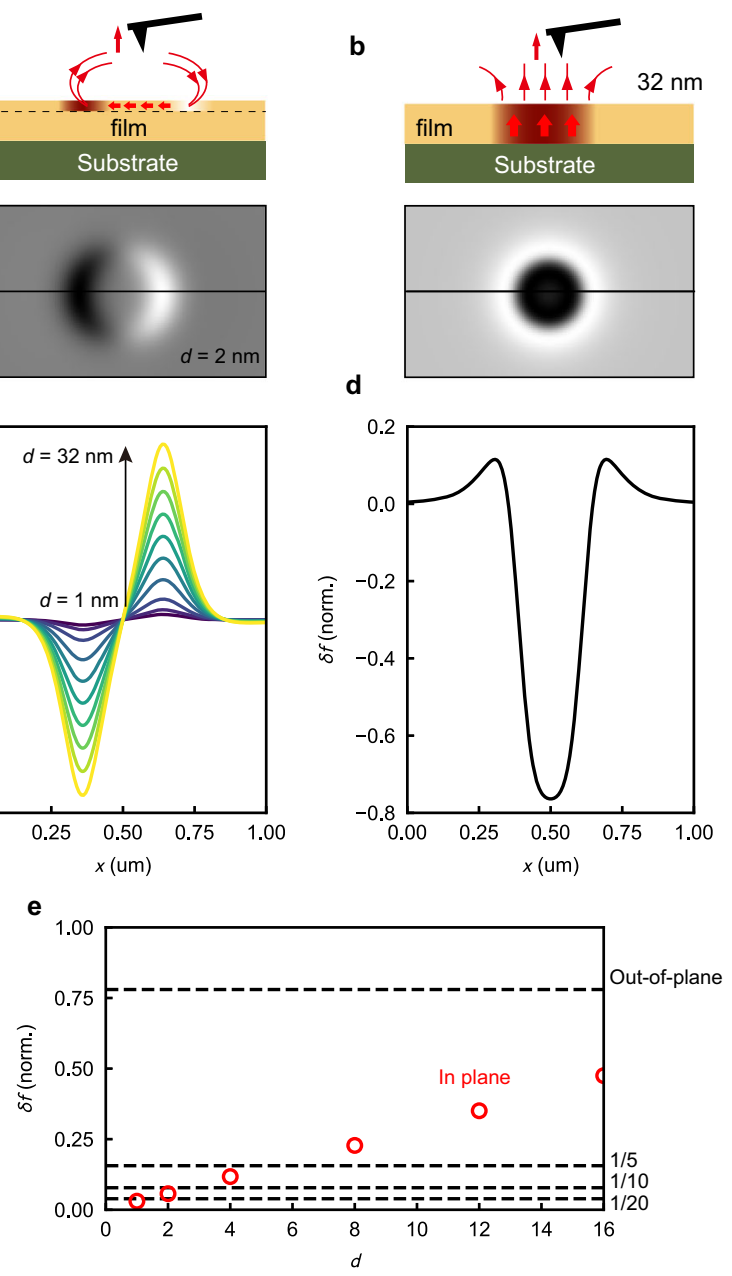

d

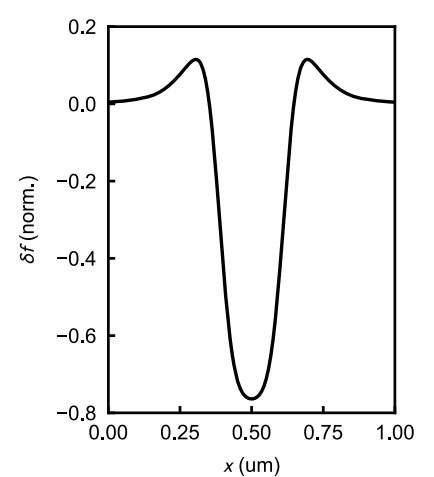

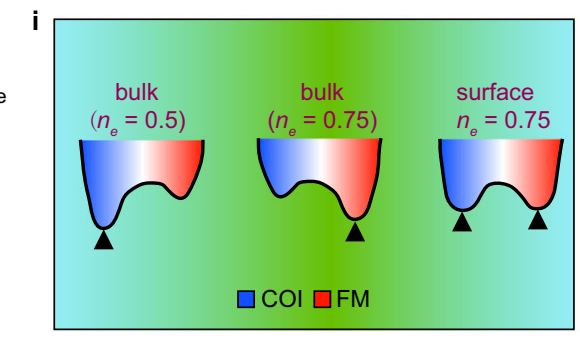

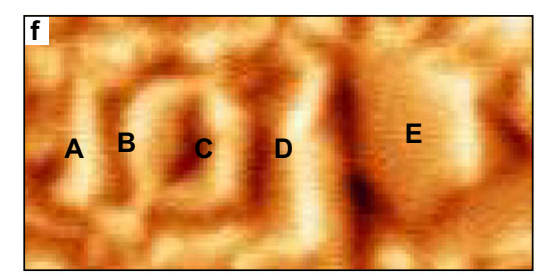

g
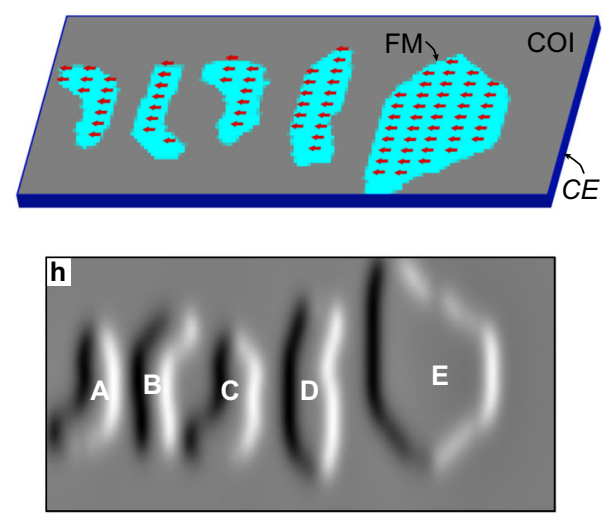

Fig. 5 Simulation of the magnetic images. a-b Simulated magnetic images of in-plane and out-of-plane magnetic domains. Size of simulated image: $1 \times 0.6 \mu \mathrm{m}^{2}$. The domain with $2 \mathrm{~nm}$ thickness is shown in a. Both domains have a diameter of $300 \mathrm{~nm}$. c The thickness dependence of the profiles along the horizontal line across the center of domains. $\mathbf{d}$ Profile along the line in $\mathbf{b}$. All the profiles in $\mathbf{c}$ and $\mathbf{d}$ are normalized by the same factor. e The thickness dependence of the contrast magnitude of in-plane magnetic domains (red open circles). The dashed line is the contrast of out-of-plane magnetic domains divided by a factor of 1, 5, 10, and 20. $\mathbf{f}$ A zoom-in of the surface phase separation pattern. Several large FM phase domains are marked with A, B, C, D, and E. $\mathbf{g}$ Illustration of the net magnetic moments at the surface. $\mathbf{h}$ Simulated MFM image of FM phase domains (A-E) based on the magnetic moment distribution shown in $\mathbf{g}$. i Schematic showing how the ground state changes with the charge density and surface.

similar magnetic contrast and orient along the same direction, it is highly unlikely that they reside at different layers of the film or have different environments.

Hence, it is likely that they are local ferromagnetic domains located at the surface with similar thickness, which is illustrated in Fig. 5a. To deduce the thickness of the FM domains from the MFM images, we simulate the contrast of in-plane domains (Fig. 5a) residing at the surface as a function of its thickness and that of out-of-plane domains (Fig. 5b) through the whole film thickness. The magnetic signal shows an almost linear dependence on the number of layers (Fig. 5c-e). Using the magnitude ratio $\sim 20$ extracted from the experiment (Fig. 4k) yields a thickness of $\sim 1 \mathrm{~nm}$ (approximately four layers of $\mathrm{MnO}$ planes in the [011] direction) for the in-plane magnetic moments (Fig. 5e). With this thickness and a speculated distribution of the magnetic moments at the surface as in Fig. $5 \mathrm{~g}$, we can then simulate the magnetic image of the several large FM phase domains in Fig. 5f, h.

Though in the original simulation in ref. ${ }^{33}$, the simplest (001) terminated surface is considered, the results can in principle be extended to other polar terminations, including the current case, (011) termination. It is worth emphasizing that, the interior of the film at this doping level $\left(n_{\mathrm{e}}=0.75\right)$ would show an FM phase.
Hence, the surface PS is not driven by the charge density deviation. It is the surface with inversion symmetry breaking that alters the free energy landscape, which eventually leads to the phase-separated state (Fig. 5i). The suppression of AFM superexchange interaction or electron-phonon interaction at the surface is likely to be the reason ${ }^{33}$. It remains to be explored whether other surfaces of PCMO, as well as other narrowbandwidth half-doped manganites, show similar properties. Also, the detailed structure of the film surface needs to be confirmed. For instance, the model assumes that the surface has intact oxygen octahedra and no defects, but the real surface structure of (011) termination should be more complicated. The influence of defects at the surface, which would change the charge density, also needs to be taken into account.

Below we summarize the unique features of the surface PS compared with the conventional PS in the bulk of other manganites: (1) even though both surface and bulk PS are a consequence of competing interactions with similar energies, surface PS arises in the system with the strongest AFM correlation, and it is only present when the bulk is in the CE phase. (2) The FM phase domains show much higher anisotropy than the bulk domains. The magnetic moments remain in the in-plane direction 
([100] axis) even in a field of $12 \mathrm{~T}$. As far as we know, this is the largest known anisotropy. It probably originates from the superexchange interaction between magnetic moments in the surface FM phase and bulk CE phase ${ }^{45}$, which would also result in an enhancement of exchange bias ${ }^{46,47}$. This suggests a route of achieving high magnetic anisotropy through the pinning effect in PS systems. (3) The vastly different patterns seen after a fieldsweep cycle indicate the magnetic domains are not due to static impurities or strain. However, these domains are pinned in different thermal cycles. This pinning effect, which is only observed in thermal cycles, remains unclear.

Nevertheless, they share some common features. The textures of the surface PS do not change with the magnetic field until the COI phase domains are melted ${ }^{39}$, which is similar to other PS systems ${ }^{39,48}$. The COI phase domains at the surface have a similar melting magnetic field as the bulk of the film.

To conclude, we revealed how the $C E$ phase is developed and its surface state in a PCMO thin film through high-field magnetic imaging. The real-space microscopic images clearly show a large suppression of the local magnetic susceptibility accompanying the charge-ordering transition at $T_{\mathrm{CO}}$, whereas no obvious change is observed at $T_{\mathrm{N}}$. These insights would help clarify the controversy regarding the nature of the phase transitions in various strongly correlated oxides. We also showed the evidence of phase separation at the surface in the $C E$ phase regime, where the ferromagnetic phase domains show very high magnetic anisotropy, confirming the ferromagnetic tendency at the surface in the $C E$ phase. These results would enrich our understanding of phase separation and spark the interest in exploring unconventional surface states in strongly correlated systems.

\section{METHODS}

\section{Magnetic force microscopy}

The description of the 20 T MFM and the detailed explanation of the image contrast formation can be found in refs. ${ }^{37,39}$. It uses the frequencymodulated mode to image the stray field from the sample, and the contrast $(\delta f)$ is proportional to the second derivative of $B_{\mathrm{z}}\left(\delta f \propto \frac{\partial^{2} B_{z}}{\partial z^{2}}\right)$. When the magnetic moments of both the tip and sample are all aligned with the external magnetic field, the darker area (with a larger negative $\delta f$ ) is then due to a larger attractive force. The lift height is $\sim 100 \mathrm{~nm}$ for all the images. WSXM is used to analyze the MFM images ${ }^{49}$.

\section{PCMO film deposition and characterization}

The details regarding the deposition and structural characterization of the thin film have been described elsewere ${ }^{35-37}$. The resistivity was measured using a physical property measurement system from Quantum Design. The magnetization was measured in a superconducting quantum interference device from Quantum Design.

\section{Magnetic image simulation}

Mumax $3^{50}$ is used to perform the micromagnetic simulations presented in this work. As it can simulate MFM images by calculating the same quantity, i.e., the second derivative of $B_{z}$ on $z$ ( $z$ is in the out-of-plane direction) ${ }^{50}$, we can compare these simulated images with the measured images directly. We use a grid of $128 \times 128 \times 32$ pixels with an actual size of $1 \mu \mathrm{m} \times 1 \mu \mathrm{m} \times$ $32 \mathrm{~nm}$. The domain diameter is $300 \mathrm{~nm}$. The saturation magnetization and exchange stiffness are $6 \times 10^{5} \mathrm{~A} / \mathrm{m}$ and $10^{-12} \mathrm{~J} / \mathrm{m}$, respectively. The tip is set as a magnetic dipole. The tip lift height is set to $150 \mathrm{~nm}$ as it shows the profile which matches the best with the experiment. The magnetic images of in-plane domains with the thickness of $1,2,4,8,12,16,20,24,28$, and $32 \mathrm{~nm}$, and out-of-planes with $32 \mathrm{~nm}$ thickness have been simulated. The robustness of the simulation can be seen in Supplementary Figures 6 and 7 .

\section{DATA AVAILABILITY}

The data that support the findings of this study are available from the corresponding authors upon reasonable request.

\section{CODE AVAILABILITY}

The Mumax 3 codes used in this study are available from the corresponding authors upon reasonable request.

Received: 28 January 2021; Accepted: 19 April 2021;

Published online: 03 June 2021

\section{REFERENCES}

1. Dagotto, E. Complexity in strongly correlated electronic systems. Science 309, 257-262 (2005).

2. Goodenough, J. B. Theory of the role of covalence in the perovskite-type manganites [La, M(II)] $\mathrm{MnO}_{3}$. Phys. Rev. 100, 564-573 (1955).

3. Lees, M. R., Barratt, J., Balakrishnan, G., Mc, K. P. D. \& Yethiraj, M. Influence of charge and magnetic ordering on the insulator-metal transition in $\operatorname{Pr}_{1-\mathrm{x}} \mathrm{Ca}_{\mathrm{x}} \mathrm{MnO}_{3}$ Phys. Rev. B 52, 14303-14307 (1995).

4. Tomioka, Y., Asamitsu, A., Kuwahara, H., Moritomo, Y. \& Tokura, Y. Magnetic-fieldinduced metal-insulator phenomena in $\mathrm{Pr}_{1-\mathrm{x}} \mathrm{Ca}_{\mathrm{x}} \mathrm{MnO}_{3}$ with controlled chargeordering instability. Phys. Rev. B 53, R1689-R1692 (1996).

5. Daoud-Aladine, A., Rodriguez-Carvajal, J., Pinsard-Gaudart, L., Fernandez-Diaz, M. T. \& Revcolevschi, A. Zener polaron ordering in half-doped manganites. Phys. Rev. Lett. 89, 097205 (2002).

6. Tomioka, Y. et al. Magnetic-field-induced metal-insulator transition in perovskitetype manganese oxides. Phys. B Condens. Matter 237-238, 6-10 (1997).

7. Millange, F., Brion, S. D. \& Chouteau, G. Charge, orbital, and magnetic order in $\mathrm{Nd}_{0.5} \mathrm{Ca}_{0.5} \mathrm{MnO}_{3}$. Phys. Rev. B 62, 5619-5626 (2000).

8. Moritomo, Y., Tomioka, Y., Asamitsu, A., Tokura, Y. \& Matsui, Y. Magnetic and electronic properties in hole-doped manganese oxides with layered structures: $\mathrm{La}_{1-\mathrm{x}} \mathrm{Sr}_{1+\mathrm{x}} \mathrm{MnO}_{4}$. Phys. Rev. B 51, 3297-3300 (1995).

9. Dagotto, E. Nanoscale phase separation and colossal magnetoresistance: the physics of manganites and related compounds. (Springer Science \& Business Media, 2013).

10. van den Brink, J., Khaliullin, G. \& Khomskii, D. Charge and orbital order in halfdoped manganites. Phys. Rev. Lett. 83, 5118-5121 (1999).

11. Jirák, Z. et al. Magnetism and charge ordering in $\operatorname{Pr}_{0.5} \mathrm{Ca}_{\mathrm{x}} \mathrm{Sr}_{0.5-\mathrm{x}} \mathrm{MnO}_{3}(\mathrm{x}=0.09$ and 0.5). Phys. Rev. B 61, 1181-1188 (2000).

12. Tokunaga, M., Miura, N., Tomioka, Y. \& Tokura, Y. High-magnetic-field study of the phase transitions of $\mathrm{R}_{1-\mathrm{x}} \mathrm{Ca}_{\mathrm{x}} \mathrm{MnO}_{3}(\mathrm{R}=\mathrm{Pr}, \mathrm{Nd})$. Phys. Rev. B 57, 5259-5264 (1998).

13. Marumoto, K., Soda, K., Kuroda, S.-I. \& Moritomo, Y. ESR Studies of Layered-Perovskite Manganites $\mathrm{R}_{0.5} \mathrm{Sr}_{1.5} \mathrm{MnO}_{4}(\mathrm{R}=\mathrm{La}, \mathrm{Nd})$. J. Phys. Soc. Jpn. 72, 582-587 (2003).

14. Ye, F. et al. Electronically smecticlike liquid-crystal phase in a nearly half-doped manganite. Phys. Rev. B 72, 212404 (2005).

15. Senff, D. et al. Melting of magnetic correlations in charge-orbital ordered $\mathrm{La}_{1 / 2} \mathrm{Sr}_{3 /}$ ${ }_{2} \mathrm{MnO}_{4}$ : competition of ferromagnetic and antiferromagnetic states. Phys. Rev. $B$ 77, 184413 (2008).

16. Jooss, C. et al. Polaron melting and ordering as key mechanisms for colossal resistance effects in manganites. Proc. Natl Acad. Sci. USA 104, 13597-13602 (2007)

17. Wu, L., Klie, R. F., Zhu, Y. \& Jooss, C. Experimental confirmation of Zener-polarontype charge and orbital ordering in $\mathrm{Pr}_{1-\mathrm{x}} \mathrm{Ca}_{\mathrm{x}} \mathrm{MnO}_{3}$. Phys. Rev. B 76, 174210 (2007).

18. Massee, F. et al. Bilayer manganites reveal polarons in the midst of a metallic breakdown. Nat. Phys. 7, 978-982 (2011).

19. Giovannetti, G., Kumar, S., van den Brink, J. \& Picozzi, S. Magnetically induced electronic ferroelectricity in half-doped manganites. Phys. Rev. Lett. 103, 037601 (2009).

20. Lopes, A. M. et al. New phase transition in the $\operatorname{Pr}_{1-x} \mathrm{Ca}_{x} \mathrm{MnO}_{3}$ system: evidence for electrical polarization in charge ordered manganites. Phys. Rev. Lett. 100, 155702 (2008).

21. Zou, T., Wang, F., Liu, Y., Yan, L.-Q. \& Sun, Y. Multiferroicity and magnetoelectric coupling in half-doped manganite $\mathrm{La}_{0.5} \mathrm{Ca}_{0.5} \mathrm{MnO}_{3}$. Appl. Phys. Lett. 97, 092501 (2010).

22. Garcia-Fernandez, M. et al. Orbital order at $\mathrm{Mn}$ and $\mathrm{O}$ sites and absence of Zener polaron formation in manganites. Phys. Rev. Lett. 103, 097205 (2009).

23. Grenier, $\mathrm{S}$. et al. Resonant $\mathrm{x}$-ray diffraction of the magnetoresistant perovskite $\mathrm{Pr}_{0.6} \mathrm{Ca}_{0.4} \mathrm{MnO}_{3}$. Phys. Rev. B 69, 134419 (2004).

24. Subias, $\mathrm{G}$. et al. Checkerboard-ordered pattern of $\mathrm{Bi}_{0.5} \mathrm{Sr}_{0.5} \mathrm{MnO}_{3}$ low-temperature phase probed by x-ray resonant scattering. Phys. Rev. B 73, 205107 (2006).

25. Senff, D. et al. Spin-wave dispersion in orbitally ordered $\mathrm{La}_{1 / 2} \mathrm{Sr}_{3 / 2} \mathrm{MnO}_{4}$. Phys. Rev. Lett. 96, 257201 (2006)

26. Herrero-Martin, J., Garcia, J., Subias, G., Blasco, J. \& Sanchez, M. C. Structural origin of dipole $x$-ray resonant scattering in the low-temperature phase of $\mathrm{Nd}_{0.5} \mathrm{Sr}_{0.5} \mathrm{MnO}_{3}$. Phys. Rev. B 70, 024408 (2004).

27. Johnstone, G. E., Perring, T. G., Sikora, O., Prabhakaran, D. \& Boothroyd, A. T. Ground state in a half-doped manganite distinguished by neutron spectroscopy. Phys. Rev. Lett. 109, 237202 (2012). 
28. Ewings, R. A. et al. Spin excitations used to probe the nature of exchange coupling in the magnetically ordered ground state of $\operatorname{Pr}_{0.5} \mathrm{Ca}_{0.5} \mathrm{MnO}_{3}$. Phys. Rev. $B$ 94, 014405 (2016).

29. Coey, M. Charge-ordering in oxides. Nature 430, 155-157 (2004).

30. Trokiner, A., Yakubovskii, A., Verkhovskii, S., Gerashenko, A. \& Khomskii, D. ${ }^{17} \mathrm{O}$ NMR as a conclusive probe of charge-ordering models in half-doped manganites. Phys. Rev. B 74, 092403 (2006).

31. Rao, S. S. \& Bhat, S. V. Probing the existing magnetic phases in $\operatorname{Pr}_{0.5} \mathrm{Ca}_{0.5} \mathrm{MnO}_{3}$ (PCMO) nanowires and nanoparticles: magnetization and magneto-transport investigations. J. Phys. Condens. Matter 22, 116004 (2010).

32. Zhang, T., Wang, X. P. \& Fang, Q. F. Exchange bias effect and suppression of charge ordering induced by a surface phase separation in $\mathrm{La}_{0.25} \mathrm{Ca}_{0.75} \mathrm{MnO}_{3}$ nanowires. J. Phys. Chem. C. 114, 11796-11800 (2010).

33. Dong, S., Yu, R., Yunoki, S., Liu, J. M. \& Dagotto, E. Ferromagnetic tendency at the surface of CE-type charge-ordered manganites. Phys. Rev. B 78, 064414 (2008).

34. Valencia, S. et al. Enhancement of spin-orbit coupling at manganite surfaces. Phys. Rev. B 98, 115142 (2018).

35. Okuyama, D. et al. Epitaxial-strain effect on charge/orbital order in $\operatorname{Pr}_{0.5} \mathrm{Ca}_{0.5} \mathrm{MnO}_{3}$ films. Appl. Phys. Lett. 95, 152502 (2009).

36. Wadati, $H$. et al. Revealing orbital and magnetic phase transitions in $\mathrm{Pr}_{0.5} \mathrm{Ca}_{0.5} \mathrm{MnO}_{3}$ epitaxial thin films by resonant soft x-ray scattering. N. J. Phys. 16, 033006 (2014)

37. Sheng, Z. et al. Visualization of electronic multiple ordering and its dynamics in high magnetic field: evidence of electronic multiple ordering crystals. ACS Appl. Mater. Interfaces 10, 20136-20141 (2018).

38. Zhou, H., Wang, Z., Hou, Y. \& Lu, Q. A compact high field magnetic force microscope. Ultramicroscopy 147, 133-136 (2014).

39. Zhou, H. et al. Evolution and control of the phase competition morphology in a manganite film. Nat. Commun. 6, 8980 (2015).

40. Israel, C., Wu, W. \& Lozanne, A. D. High-field magnetic force microscopy as susceptibility imaging. Appl. Phys. Lett. 89, 032502 (2006).

41. Wang, Z.-H., Lebedev, O. I., Van Tendeloo, G., Cristiani, G. \& Habermeier, H. U. Crosshatching on $\mathrm{La}_{0.5} \mathrm{Ca}_{0.5} \mathrm{MnO}_{3}$ ultrathin films epitaxially grown on $\mathrm{SrTiO}_{3}(100)$. Phys. Rev. B 77, 115330 (2008).

42. Solovyev, I. V. Charge ordering due to magnetic symmetry breaking. Phys. Rev. Lett. 91, 177201 (2003).

43. Bao, W., Axe, J. D., Chen, C. H. \& Cheong, S. W. Impact of charge ordering on magnetic correlations in perovskite (Bi,Ca) $\mathrm{MnO}_{3}$. Phys. Rev. Lett. 78, 543-546 (1997).

44. Chen, C. H., Cheong, S. W. \& Cooper, A. S. Charge modulations in $\mathrm{La}_{2-x} \mathrm{Sr}_{x} \mathrm{NiO}_{4+y}$ : ordering of polarons. Phys. Rev. Lett. 71, 2461-2464 (1993).

45. Meiklejohn, W. H. \& Bean, C. P. New magnetic anisotropy. Phys. Rev. 105, 904-913 (1957).

46. Giri, S. K., Yusuf, S. M., Mukadam, M. D. \& Nath, T. K. Enhanced exchange bias effect in size modulated $\mathrm{Sm}_{0.5} \mathrm{Ca}_{0.5} \mathrm{MnO}_{3}$ phase separated manganite. J. Appl. Phys. 115, 093906 (2014).

47. Stamps, R. L. Mechanisms for exchange bias. J. Phys. D. 33, R247-R268 (2000)

48. Lai, K. et al. Mesoscopic percolating resistance network in a strained manganite thin film. Science 329, 190-193 (2010).

49. Horcas, I. et al. WSXM: a software for scanning probe microscopy and a tool for nanotechnology. Rev. Sci. Instrum. 78, 013705 (2007).

50. Vansteenkiste, A. et al. The design and verification of MuMax3. AIP Adv. 4, 107133 (2014).

\section{ACKNOWLEDGEMENTS}

We thank professor Shuai Dong, professor Fusheng Ma, and Dr. Jonathan C. Leiner for helpful discussions. We gratefully acknowledge financial support from the National
Key Research and Development Program of China (grant nos. 2017YFA0402903, 2017YFA0303603, 2016YFA0401003, and 2016YFA0401803), the National Natural Science Foundation of China (NSFC; grant nos. U1932216, 51627901, U2032218, 11574316, 21505139, 11474263, U1432251, 11374278, U1632160, and 11704384), Maintenance and Renovation Project for CAS (grant no. DSS-WXGZ-2019-0011), the Key Research Program of Frontier Sciences, CAS (grant no. QYZDB-SSW-SLH011), the Instrument Developing Project of the Chinese Academy of Sciences (grant no. YZ201423), Hefei Science Center CAS (grant no. 2020HSC-UE014), High Magnetic Field Laboratory of Anhui Province, Anhui Lab of Adv. Photon Sci. and Tech., the Chinese Academy of Science Scientific Research Equipment (grant no. YZ201628), and the One Thousand Youth Talents Program of China. M.N. was supported by Japan Science and Technology (JST), PRESTO (JPMJPR16R5).

\section{AUTHOR CONTRIBUTIONS}

H.Z., Z.S., and Q.L. conceived the project. H.Z. and Q.F. conducted the MFM measurements with assistance from Y.H. Z.S. made the sample and measured the transport with the help of M.N., Y.T., and M.K. Q.F. performed the magnetization measurement. H.Z. performed the micromagnetic simulation. H.Z., Z.S., and Q.L. analyzed the data. H.Z. wrote the manuscript under the supervision of Z.S. and Q.L. with input from all the other authors.

\section{COMPETING INTERESTS}

The authors declare no competing interests.

\section{ADDITIONAL INFORMATION}

Supplementary information The online version contains supplementary material available at https://doi.org/10.1038/s41535-021-00353-2.

Correspondence and requests for materials should be addressed to H.Z., Z.S. or Q.L.

Reprints and permission information is available at http://www.nature.com/ reprints

Publisher's note Springer Nature remains neutral with regard to jurisdictional claims in published maps and institutional affiliations.

Open Access This article is licensed under a Creative Commons Attribution 4.0 International License, which permits use, sharing, adaptation, distribution and reproduction in any medium or format, as long as you give appropriate credit to the original author(s) and the source, provide a link to the Creative Commons license, and indicate if changes were made. The images or other third party material in this article are included in the article's Creative Commons license, unless indicated otherwise in a credit line to the material. If material is not included in the article's Creative Commons license and your intended use is not permitted by statutory regulation or exceeds the permitted use, you will need to obtain permission directly from the copyright holder. To view a copy of this license, visit http://creativecommons. org/licenses/by/4.0/.

(c) The Author(s) 2021 